\title{
steady-state Microbunching in a Storage Ring for Generating Coherent Radiation
}

\author{
Daniel F. Ratner ${ }^{1}$ and Alexander W. Chao ${ }^{2}$ \\ ${ }^{1}$ Department of Applied Physics, Stanford University, Stanford, CA 94305 \\ ${ }^{2}$ SLAC National Accelerator Laboratory, Stanford University, Stanford, CA 94309
}

\begin{abstract}
Synchrotrons and storage rings deliver radiation across the electromagnetic spectrum at high repetition rates, and free electron lasers (FELs) produce radiation pulses with high peak brightness. However, at present few light sources can generate both high repetition rate and high brightness outside the optical range. We propose to create steady-state microbunching (SSMB) in a storage ring to produce coherent radiation at a high repetition rate or in continuous wave $(\mathrm{CW})$ mode. In this paper we describe a general mechanism for producing SSMB and give sample parameters for EUV lithography and sub-millimeter sources. We also describe a similar arrangement to produce two pulses with variable spacing for pump-probe experiments. With technological advances, SSMB could reach the soft X-ray range $(<10 \mathrm{~nm})$.
\end{abstract}

\section{INTRODUCTION}

In a radiation source driven by coherent electrons, particles group into microbunches spaced at the wavelength of the desired light. The resulting coherent light can be orders of magnitude brighter than that of an equivalent incoherent source. The FEL process, for example, turns a constant stream of electrons into a series of microbunches, which then radiate coherently.

For high average power light sources, duty-cycle shares the stage with coherence. Linac driven FELs use each electron pulse once, leading to low duty cycles. (Energy recovery linacs reach high duty cycles by recovering the electron energy [1-3].) Storage rings, by contrast, naturally provide $\mathrm{MHz}$ repetition rates, and fully filled rings can provide $\mathrm{CW}$ radiation. However, storage rings do not generally support sustained microbunching (MB).

Using an optical or RF modulation, we propose to microbunch stored electrons during each pass through a radiator. Though the electrons may appear smeared elsewhere in the ring, the MB is permanent at the radiator, so we consider this SSMB. The result is a coherent radiation source with $\mathrm{MHz}$ to $\mathrm{CW}$ repetition rate.

In a conventional storage ring, RF 'buckets' both accelerate and trap electrons. The RF modulation accelerates electrons in front of the stable point (which then slip backward because of dispersion) while decelerating electrons behind the stable point (which then slip forward), so that all particles tend to move toward the stable point. Instead of a continuous stream of electrons, we find a train of tightly 'bunched' beamlets spaced at the $\mathrm{RF}$ wavelength. Replacing the RF with an optical laser results in a beam bunched at optical wavelengths [4].

The drawback to bucket bunching is that the output radiation is limited to the initial radiation wavelength; to produce high power radiation at a wavelength, $\lambda_{\text {out }}$, we need bunching at $\lambda_{\text {out }}$, which in turn requires high power radiation at $\lambda_{\text {out }}$. Instead, we propose to modulate with an easily available initial wavelength (e.g. optical) $\lambda_{\text {in }}$, but generate stable points distributed at either a harmonic or a multiple of $\lambda_{\text {in }}$. To distinguish harmonic or multiplied bunching from conventional RF buckets, we will refer to trapping at $\lambda_{\text {out }} \neq \lambda_{\text {in }}$ as MB. For example, seeding with $\lambda_{\text {in }}$ between $200 \mathrm{~nm}$ and $2 \mu \mathrm{m}$ (easily available from commercial sources), we can produce MB at wavelengths ranging from $\lambda_{\text {out }}=13.5 \mathrm{~nm}$ to $1 \mathrm{~mm}$. We note that single-shot versions of $\mathrm{MB}\left(\lambda_{\text {in }} \neq \lambda_{\text {out }}\right)$ are mainstays of FEL seeding $[5,6]$.

\section{SSMB MECHANISM}

\section{A. Zero-Crossing SSMB}

In general, particles bunch around stable fixed points. A particle is at a fixed point in phase space if, after $T$ turns around the ring, the particle returns to its initial coordinates; with a one turn map $M$, a fixed point exists in phase space at $X_{0}=(z, p)$ if $M^{T} X_{0}=X_{0}$. Each RF bucket contains only one fixed point per wavelength (at $z=m \lambda_{\text {in }}, p=0$, for integers $m$ ), so the standard RF bucket generates bunching, not MB.

(In all discussions, energy, $p \equiv E-E_{\text {beam }}$, and position, $z$, are given relative to the ideal electron defined as $p_{I} \equiv$ $0, z_{I} \equiv 0$. We will consider the case of operation above transition. Below transition, the slippage reverses.)

As an example of $\mathrm{MB}$, we return to the particle at a zero-crossing of a sine modulation. Instead of $p=0$, we now consider a particle with a special energy $p=\Delta p$, such that the particle slips backward by $\Delta z=\lambda_{\text {in }}$ each turn due to dispersion. The particle does not return to its initial position, but because of the modulation's periodicity, the particle moves to an equivalent zero-crossing, so we still consider this to be a fixed point. At each zerocrossing, we find a set of such fixed points arrayed at energies $p=n \Delta p$, for $n=0, \pm 1, \pm 2$, etc. Fixed points with positive (negative) energies slip backward (forward) $n$ wavelengths per turn.

After a full turn, at the ring's modulation point, the microbunches stack at $z=m \lambda_{\text {in }}$, with one stack per modulation wavelength, i.e., the electrons are bunched at $\lambda_{\text {in }}$. However, after a fraction, $1 / H$, of a turn, the fixed points have slipped only $\Delta z=n \lambda_{\text {in }} / H$, and the particles are spaced at the $H^{\text {th }}$ harmonic of $\lambda_{\text {in }}$, i.e. are 
microbunched at $\lambda_{\text {out }}=\lambda_{\text {in }} / H$ (Fig. 1).

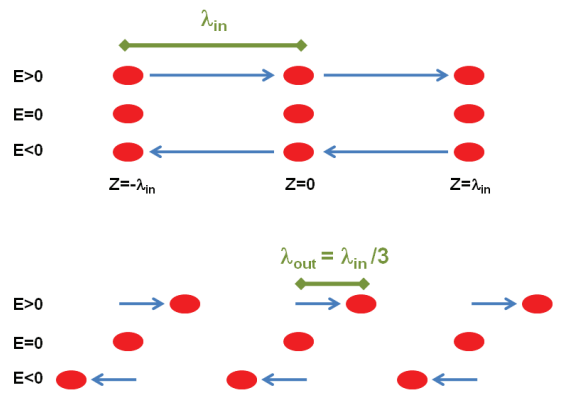

FIG. 1. An illustration of harmonic SSMB for $H=3$. At top, we show particles in phase space at the modulator. Each turn around the ring, particles slip forward or backward from dispersion, but the distribution is stationary for a periodic modulation. At bottom, at an intermediate point in the ring ( $1 / H$ the way around), the microbunches are spaced by $\lambda_{\text {in }}$ $/ H$, i.e. the beam is microbunched with $\lambda_{\text {out }}=\lambda_{\text {in }} / H$.

\section{B. Double Modulations}

For a general account of the SSMB principle we describe a two-stage system; multiple modulation and dispersive regions improve control of phase space at a small cost in complexity (Fig. 2). We break each turn into 4 steps:

$$
\begin{aligned}
z_{1}=z_{0}, & p_{1}=p_{0}+F_{a}\left(z_{0}\right) \\
z_{2}=z_{1}+R_{56}^{(a)} p_{1}, & p_{2}=p_{1} \\
z_{3}=z_{2}, & p_{3}=p_{2}+F_{b}\left(z_{2}\right) \\
z_{4}=z_{3}+R_{56}^{(b)} p_{3}, & p_{4}=p_{3} .
\end{aligned}
$$

In step 1, we apply a modulation to the beam energy, $F_{a}\left(z_{0}\right)$. In step 2 , dispersion $R_{56}^{(a)}$ converts the change in energy to a change in position. We then repeat with a second modulation, $F_{b}\left(z_{2}\right)$, and dispersive section, $R_{56}^{(b)}$. We find single turn $(T=1)$ fixed points whenever we satisfy the slippage condition:

$$
\begin{aligned}
& \Delta z_{\text {onepass }}=R_{56}^{(a)} p_{1}+R_{56}^{(b)} p_{3}=n \lambda_{\text {in }} \\
& \Delta p_{\text {onepass }}=F_{a}\left(z_{0}\right)+F_{b}\left(z_{2}\right)=0
\end{aligned}
$$

for $n=0, \pm 1, \pm 2$, etc.

Most generally, we are looking for any combination of $R_{56}^{(a)}, R_{56}^{(b)}, F_{a}, F_{b}$ such that the resulting fixed points have a clean, periodic structure. In our previous example (fixed points at the modulation zero-crossing), we set $F_{a}\left(z_{0}\right)=F_{b}\left(z_{2}\right)=0$ and $R_{56}^{(a)}=R_{56}^{(b)}$. However, other manipulations are possible. For example, setting $F_{a}\left(z_{0}\right)=-F_{b}\left(z_{2}\right)$, so that the two modulations cancel, also produces harmonic SSMB. (Canceling modulations produce clean harmonic structure only for sawtooth waveforms, $F_{a, b}=V_{a, b} \bmod _{\lambda} z$.) steady-state echo enabled harmonic generation may be capable of driving SSMB at high harmonics [6].

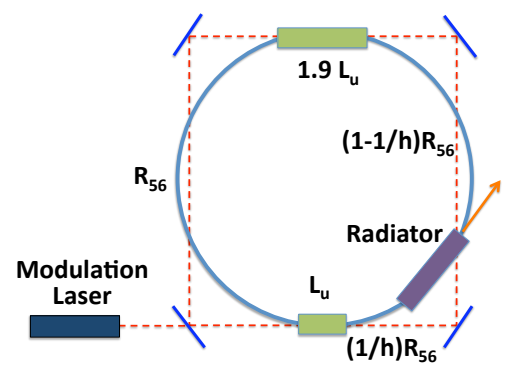

FIG. 2. Example schematic for a two-stage system. A laser cavity and two undulators of length $L_{u}$ and $1.9 L_{u}$ modulate the electron beam at opposite ends of a storage ring. SSMB from the modulation and dispersion produces coherent light in a radiator. $\mathrm{RF}$ modules could replace the laser modulation to produce long wavelengths, and an additional radiator could be placed at the same distance in front of $L_{u}$.

\section{Analysis of Linearized Modulation}

For simulations we consider modulations $F_{a, b}=$ $V_{a, b} \sin (k z)$. To study the zero-crossing fixed points analytically, we linearize the modulation, $F_{a, b}(z) \approx h_{a, b} z$, with $h_{a, b} \equiv V_{a, b} / k$, and write the one turn map as a matrix

$$
\begin{aligned}
M & =\left(\begin{array}{cc}
1 & R_{56}^{(b)} \\
0 & 1
\end{array}\right)\left(\begin{array}{cc}
1 & 0 \\
h_{b} & 1
\end{array}\right)\left(\begin{array}{cc}
1 & R_{56}^{(a)} \\
0 & 1
\end{array}\right)\left(\begin{array}{cc}
1 & 0 \\
h_{a} & 1
\end{array}\right) \\
& =\left(\begin{array}{cc}
1+s(2+(1+s) \eta) & \frac{s}{h_{a}}(2+s \eta) \\
h_{a}(1+(1+s) \eta) & 1+s \eta
\end{array}\right),
\end{aligned}
$$

with $R_{56}^{(a)}=R_{56}^{(b)} \equiv s / h_{a}$ and $\eta \equiv h_{b} / h_{a}$. The stability condition, $|\operatorname{Tr} M|<2$, constrains

$$
-4<2(s+s \eta)+s^{2} \eta<0 .
$$

We also write down an equilibrium bunch length using the Courant-Snyder parameters, $\left\langle z^{2}\right\rangle=\beta \varepsilon$ and $\left\langle\delta^{2}\right\rangle=$ $\gamma \varepsilon$, yielding

$$
\left\langle z^{2}\right\rangle=-s \frac{2+s \eta}{(1+\eta+s \eta)} \frac{\left\langle\delta^{2}\right\rangle}{h_{a}^{2}},
$$

suggesting that $s \eta \sim-2$ can substantially reduce the island size, increasing the maximum possible harmonic. In reality, energy changes from damping and quantum excitation, combined with dispersion, will increase $\left\langle z^{2}\right\rangle$. However, in simulations we do observe moderate bunch compression as $s \eta \rightarrow-2$. 


\section{Long Wavelength Beating}

It is possible to generate MB with $\lambda_{\text {out }} \gg \lambda_{\text {in }}$ by using different input wavelengths, $\lambda_{\text {in } 2}=[b /(b-1)] \lambda_{\text {in } 1}$ [7]. The wavelength beating results in periodicity at $\lambda_{\text {out }}=b \lambda_{\text {in } 1}$, with the potential to set $b \gg 1$. We can drive SSMB from frequency beating in two ways. For a double modulation (separate modulation and dispersive regions for each wavelength), stable fixed points survive only where the phases overlap constructively, resulting in sets of fixed points separated by the beat wavelength, $\lambda_{\text {out }}$. For a single modulation (beating the lasers prior to modulation, or equivalently using a single dispersive region), stable fixed points survive across $\lambda_{\text {out }}$. However, if we rely on the modulation to replace the synchrotron radiation energy loss, $\delta$, fixed points will only survive where the combined modulation, $V_{a} \cos \left(k_{a} z\right)+V_{b} \cos \left(k_{b} z\right)$, is larger than $\delta$. For $\delta \lesssim V_{a}+V_{b}$, stable fixed points remain only where the phases overlap, near $\bmod _{\lambda_{\text {out }}} z=0$, again resulting in SSMB at the beat wavelength, $\lambda_{\text {out }}$ (Fig. 3).
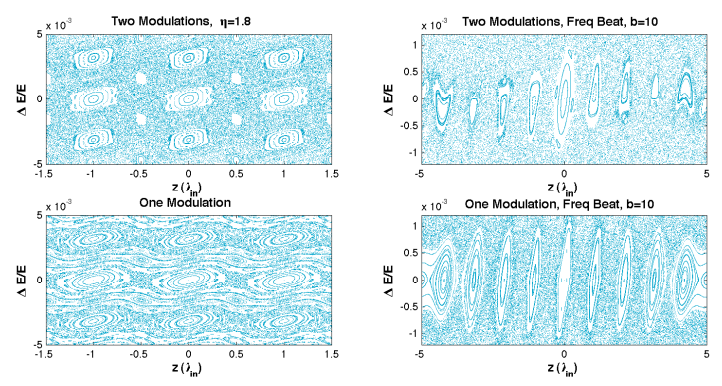

FIG. 3. Phase space for harmonic SSMB at left and frequency beating at right (with $b=10$ ), with two-stage modulations on top and one-stage modulations below. For harmonic SSMB, two-stage modulations provide a cleaner phase space with shorter stable regions. For frequency beating, the one-stage phase space has stable islands throughout $\lambda_{\text {out }}=b \lambda_{\text {in }}$, but radiative energy loss removes the side islands.

\section{RADIATION SOURCE FROM SSMB}

\section{A. EUV Source}

For each turn around the ring, the SSMB beam passes through a radiator (e.g. an undulator). Matching the $\mathrm{MB}$ wavelength to the resonant wavelength of an undulator strongly amplifies the radiation brightness. As a first example, we consider the feasibility of SSMB at the EUV lithography standard, $13.5 \mathrm{~nm}$. To produce a stable, high power CW modulation, we overlay the storage ring with a laser cavity and two undulators, with a third undulator for output (Fig. 2). In general, a laser modulation does not involve any average energy transfer. However, in the absence of an RF bucket, the modulation process replaces the energy loss from radiative energy loss.
We illustrate the concept with a simulation that tracks the 4-D particle coordinates $\left(z, p, x, x^{\prime}\right)$ through a large number of passes around the ring. The simulation includes both energy loss $(\delta)$ and quantum fluctuations $(\epsilon)$ from synchrotron radiation, as well as first and second order momentum compaction $\left(R_{56}, T_{566}\right)$ and transverse transport elements $\left(R_{51}, R_{52}\right)$. We model jitter errors by shifting the modulation relative to the particles on a turn-by-turn basis. We assume an initially flat-top longitudinal distribution with Gaussian transverse profile of radius $\sigma_{x}, \sigma_{x^{\prime}}$, though we note the two synchrotron radiation terms, $\delta$ and $\epsilon$, will determine the equilibrium unmodulated energy spread $\left(\sigma_{\delta}\right)$. Periodic boundary conditions allow for slippage across many wavelengths.

For a $13.5 \mathrm{~nm}$ source, we simulate a $500 \mathrm{MeV}$ ring, modulated at $\lambda_{\text {in }}=200 \mu \mathrm{m}$ and look for SSMB at the 15th harmonic (Tab. I). To maintain the short wavelength SSMB we require a large energy aperture $( \pm 6 \%)$ and a laser cavity with a strong $50 \mathrm{MW}$ stored power, though only with a few $\mathrm{kW}$ of $\mathrm{CW}$ input power. Stability of the laser is assumed to be $0.005^{\circ}$ in phase and $0.005 \%$ in amplitude. Choosing $\eta=1.9$, after 10 million turns we then observe trapping of $\sim 15 \%$ of the particles with strong SSMB (Fig. 4). (A large ring with weak bends may permit decreased laser power and energy aperture, but we have not explored ring optimization.)
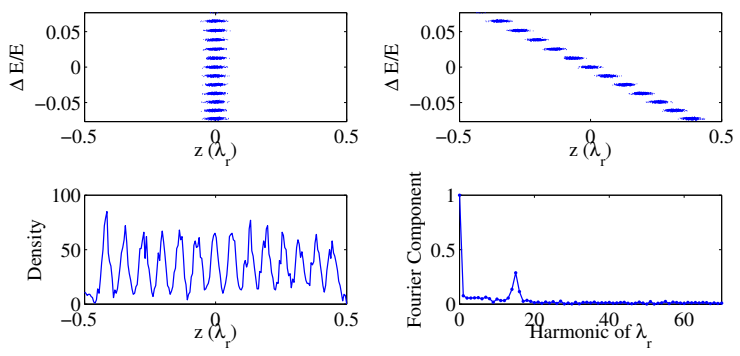

FIG. 4. Simulation of a two-stage manipulation to produce SSMB. After 10 million revolutions, we find bunches stacked at steady-state in energy space (top left). After an additional dispersive section of $R_{56} / 15$, we find SSMB at the 15 th harmonic (top right). The density profile is shown bottom left, with the Fourier transform bottom right.

\section{B. THz and Pump Probe Sources}

We also provide two illustrations of long wavelength SSMB. Rings operating with low momentum compaction factors have produced coherent radiation from isolated, short pulses with $\sigma_{z}<\lambda_{\text {out }}[8,12]$. With a coherent train of pulses from frequency beating SSMB, we propose to generate high average power $\mathrm{THz}$ radiation. As an example, we simulate $\lambda_{\text {in } 1}=2 \mu \mathrm{m}$ and multiplying factor $b=250$ for a $500 \mathrm{MeV}$ ring with realistic damping and energy spread (Tab. I). With laser stability of $0.01^{\circ}$ in phase and $.01 \%$ in amplitude, we find strong bunching at 
TABLE I. Example Parameters

\begin{tabular}{|l|c|c|c|}
\hline & EUV & THz & Pump-probe \\
\hline Rad. wavelength $\left(\lambda_{\text {out }}\right)$ & $13.5 \mathrm{~nm}$ & $500 \mu \mathrm{m}$ & NA \\
\hline Seed wavelength $\left(\lambda_{\text {in }}\right)$ & $200 \mathrm{~nm}$ & $2.0,2.008 \mu \mathrm{m}$ & $1 \mathrm{~cm}$ \\
\hline Modulation amp. $\left(V_{a}\right)$ & $1 \mathrm{MeV}$ & $350 \mathrm{keV}$ & $15 \mathrm{MeV}$ \\
\hline Dispersion $\left(R_{a}+R_{b}\right)$ & $30 \mu \mathrm{m}$ & $0.9 \mathrm{~mm}$ & $0.6 \mathrm{~m}$ \\
\hline Beam energy $(E)$ & $500 \mathrm{MeV}$ & $500 \mathrm{MeV}$ & $3 \mathrm{GeV}$ \\
\hline Damp. decrement $(\delta)$ & $5 \times 10^{-6}$ & $5 \times 10^{-6}$ & $3 \times 10^{-4}$ \\
\hline Equil. $E$ spread $\left(\sigma_{\delta}\right)$ & $3 \times 10^{-4}$ & $5 \times 10^{-4}$ & $1 \times 10^{-3}$ \\
\hline
\end{tabular}

$\lambda_{\text {out }}=500 \mu \mathrm{m}$. Better laser stability would permit lower ring energy.

Alternatively, RF modulations could be used to drive pump-probe experiments, which use two pulses, separated by a variable time delay, to study dynamical systems. Using SSMB to create two microbunches, a variable dispersive strength between the modulation and the radiator would change the time delay between pulses. While not coherent, an SSMB pump-probe at a multi$\mathrm{GeV}$ ring (Tab. I) would radiate in the X-ray regime. As a side benefit, the difference in energy of the two bunches leads to slightly different radiation wavelengths. Such 'two-color' output helps users distinguish the pump and probe pulses. We note that two-color double pulses have previously been observed from $\alpha$ buckets [13].

\section{TECHNICAL CHALLENGES}

Operating modes with low momentum compaction factor produce short bunches, creating large ratios of peak current to average current. To prevent the amplified peak currents from driving longitudinal instabilities, the average currents are typically very low [8-11]. However, the SSMB process spreads particles evenly across many microbunches in each bucket, so that the ratio of peak to average current is approximately the harmonic number, $H$, or smaller (Fig. 4). We then expect the SSMB process to support relatively large average currents despite the small momentum compaction factor.

SSMB is relatively immune to static errors in laser phase $(\phi)$, modulation amplitude $(V)$, and dispersion
$\left(R_{56}\right)$ between the two stages. For steady-state solutions, static errors only shift the equilibrium fixed points. From the EUV simulation we find $\Delta \phi<5^{\circ}, \Delta V<5 \%$, $\Delta R_{56}<1 \%$ and $T_{566} \lesssim R_{56}$ do not inhibit SSMB.

However, SSMB is potentially sensitive to stability errors, especially for long damping times. Small jitters in timing between the laser and electron bunch may accumulate and smear out the structure, requiring high phase and amplitude stability. If the laser cavity cannot achieve the required stability, the electron bunch itself could serve as the modulation source, intrinsically locking the electrons to the modulation. Self-modulation is possible for manipulations which generate bunching at $\lambda_{\text {in }}$; in Fig. 4, we see nearly $100 \%$ bunching at $\lambda_{\text {in }}$ at the modulation point (top left). However, self-modulation will increase the amplitude jitter.

The tightest constraints come from transverse transfer elements, $R_{51}, R_{52}$, which will detrap particles and lengthen the SSMB unless $R_{51} \sigma_{x}, R_{52} \sigma_{x^{\prime}} \ll \lambda_{\text {in }}, \lambda_{\text {out }}$. Particles with the largest $x, x^{\prime}$ values are lost, which may result in shrinking of $\sigma_{x}, \sigma_{x^{\prime}}$. It is interesting to note that keeping the transverse coordinates fixed (i.e., integer transverse tune, $\nu$ ) adjusts the slippage condition (Eq. 2) by $R_{56} p \rightarrow R_{56} p+R_{51} x+R_{52} x^{\prime}$. The constraints on $R_{51} \sigma_{x}$ and $R_{52} \sigma_{x^{\prime}}$ then relax, but practically it would be difficult to operate a ring close to an integer tune.

\section{CONCLUSION}

We present a mechanism for producing SSMB in a storage ring. The combination of dispersion and modulation regions produces fixed points (and thus SSMB) at harmonics or multiples of the modulation wavelength. We acknowledge that preserving fine SSMB will be challenging, and briefly discuss some potential technical requirements. A proof of principle may be possible at long wavelengths, and we will address practical design issues in future studies.

\section{ACKNOWLEDGMENTS}

We would like to thank J. Corbett, V. Dolgashev, Z. Huang, Y. Nosochkov, R. Ruth, M. Sjostrom, and D. Xiang for many helpful discussions. This work was supported by U.S. DOE Contract No. DE-AC02-76SF00515.
[1] M. Tigner, Nuovo Cimento, 37, 1228 (1965).

[2] J.M. Klopf, A. Greer, J. Gubeli, G.R. Neil, M. Shinn, T. Siggins, D. Waldman, G.P. Williams, A. Todd, V. Christina, and O. Chubar, Nucl. Instrum. Methods Phys. Res., Sect. A 582, 114 (2007).

[3] B.A. Knyazev, G.N. Kulipanov, and N.A. Vinokurov, Meas. Sci. Technol. 21054017 (2010).
[4] V. N. Litvinenko, S. F. Mikhailov, O. A. Shevchenko, and Y. Wu, Proceedings of the Particle Accelerator Conference, p. 2614 (2001)

[5] L.H. Yu, Phys. Rev. A 44, 5178 (1991).

[6] G. Stupakov, Phys. Rev. Lett. 102, 074801 (2009).

[7] S. Reiche, C. Joshi, C. Pellegrini, J.B. Rosenzweig, S.Ya. Tochitsky, and G. Shvets, Proceedings of PAC 05, 1721 (2005). 
[8] M. Abo-Bakr, J. Feikes, K. Holldack, P. Kuske, W.B. Peatman, U. Schade, G. Wüstefeld, and H.W. Hübers Phys. Rev. Lett. 90, 094801 (2003).

[9] V.K. Neil and A. Sessler, Rev. Sci. Instrum. 36, 4291965

[10] S. Heifets and G. Stupakov, Phys. Rev. ST Accel. Beams 5, 054402 (2002)

[11] M. Venturini and R. Warnock, Phys. Rev. Lett. 89, 224802 (2002).
[12] H. Hama, H. Tanaka, N. Kumagai , M. Kawai, F. Hinode, T. Muto, K. Nanbu, T. Tanaka, K. Kasamsook, K. Akiyama, and M. Yasuda, New J. Phys. 8, 292 (2006).

[13] J. B. Murphy and S. L. Kramer, Phys. Rev. Lett. 84, $5516(2000)$ 